\title{
Hybrid Wavelength Routed and Optical Packet Switched Ring Networks for the Metropolitan Area Network
}

\author{
Nord, Martin
}

Published in:

Proc. 7th IEEE International Conference on Transparent Optical Networks 2005

Link to article, DOI:

10.1109/ICTON.2005.1505801

Publication date:

2005

Document Version

Publisher's PDF, also known as Version of record

Link back to DTU Orbit

Citation (APA):

Nord, M. (2005). Hybrid Wavelength Routed and Optical Packet Switched Ring Networks for the Metropolitan Area Network. In Proc. 7th IEEE International Conference on Transparent Optical Networks 2005: ICTON 2005 (pp. 267-272). IEEE. https://doi.org/10.1109/ICTON.2005.1505801

\section{General rights}

Copyright and moral rights for the publications made accessible in the public portal are retained by the authors and/or other copyright owners and it is a condition of accessing publications that users recognise and abide by the legal requirements associated with these rights.

- Users may download and print one copy of any publication from the public portal for the purpose of private study or research.

- You may not further distribute the material or use it for any profit-making activity or commercial gain

- You may freely distribute the URL identifying the publication in the public portal 


\title{
Hybrid Wavelength Routed and Optical Packet Switched Ring Networks for the Metropolitan Area Network
}

\author{
Martin Nord ${ }^{1,2}$ \\ 1) Research Center COM, Technical University of Denmark, DK-2800 Kgs. Lyngby, Denmark \\ 2) Telenor $R \& D, N-1331$ Fornebu, Norway \\ Tel:+4748122838,e-mail:mn@com.dtu.dk
}

\begin{abstract}
Increased data traffic in the Metropolitan Area Network calls for new network architectures. This paper evaluates optical ring architectures based on optical packet switching-, wavelength routing, and hybrid combinations of the two concepts. The evaluation includes overall throughput and fairness aspects in both uniform and unbalanced traffic scenarios, for both Poisson and bursty input traffic. Simulations show a trade-off between performance and complexity. Whilst the static wavelength routed optical network is a good solutions for uniform traffic, the proposed hybrid architectures are more attractive when traffic is unbalanced.

Keywords: optical packet switching, static wavelength routed optical network, hybrid network, variable length packets, fairness, metropolitan area network.
\end{abstract}

\section{INTRODUCTION}

Future Metropolitan Area Networks (MAN) must support an increased amount of data traffic, which will make the traffic more dynamic and demanding than today [1]. The currently deployed Synchronous Digital Hierarchy $(\mathrm{SDH})$ technologies are not suitable to accommodate these changes. This future metro-gap will prevent clients in the Access Networks (ANs) from tapping into the large bandwidth available in backbone networks [1]. This challenge presents an opportunity for optical switching network architectures, if they can be bandwidth efficient, scalable and flexible, while not demanding excessive optical hardware or electronic scheduling complexity.

It is at present not clear what the QoS requirements of a future MAN network will be. In this study we address how to support the part of the traffic that tolerates a relaxed statistical PLR $\left(\sim 2 \times 10^{-3}\right.$, thus well below the $1 \%$ requirement of [2]), and a bounded delay-jitter of $8 \mu$ s (assuming a Mean Packet Durations (MPD) of $1 \mu \mathrm{s}$ ). The potential benefit of using the statistical QoS paradigm is economic use of resources by high bandwidth efficiency.

Ideally, a network with statistical QoS should support relative QoS differentiation by operating with several Classes of Service $(\mathrm{CoS})$. Moreover it should provide network-consistent CoS levels, ensuring that the performance within a $\mathrm{CoS}$ is uniform across the network. This requires a fair access to resources since e.g. if a node gets sub-average access to ring bandwidth, both high- and low CoS transfers originating at this node may have higher loss rates than low CoS transfers from other nodes. This study thus focuses on fairness aspects, since it seems to be a more fundamental issue than QoS differentiation.

The remainder of this paper is organised as follows: Section 2 describes the network topology and proposed network architectures. Section 3 presents results of dimensioning studies. Section 4 discusses these results in light of the complexity of the designs, to evaluate the network architectures. Section 5 concludes the paper.

\section{NETWORK ARCHITECTURES}

\subsection{Topology}

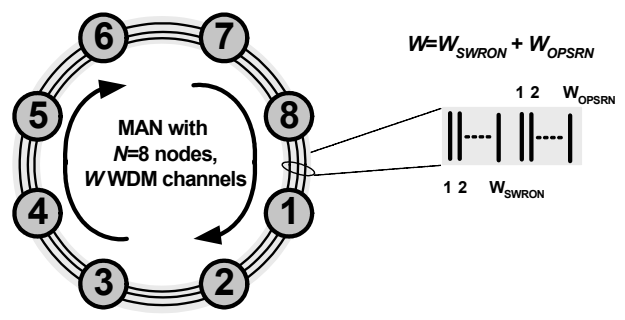

Figure 1. A unidirectional WDM ring network for the MAN.

Ring topologies are seen as an attractive alternative for the MAN [1], and are widely implemented [2]. To highlight the fundamental properties of ring networks, we investigate a single unidirectional ring. This is an important step before expanding the study to include e.g. bidirectionality and interconnection of rings, which may improve network resilience and scalability.

The work has been funded by the Research Council of Norway, and Telenor R\&D, Norway. 
Figure 2 illustrates the ring node designs for the Optical Packet Switched Ring Network (OPSRN) and Static Wavelength Routed Optical Network (SWRON) architectures that we proposed in $[3,4]$. Their principle of operation is further detailed in section 2.2 and 2.3. The main difference between the two is that the OPSRN uses a Tuneable Wavelength Converter (TWC) to switch the packets from a source node $\left(n_{S}\right)$ to a destination node $\left(n_{D}\right)$, whilst the SWRON only uses passive wavelength selective components to guide the optical signals. Both architectures use Fixed Transmitters (FT) and Fixed Receivers (FR) to insert- and to receive data.
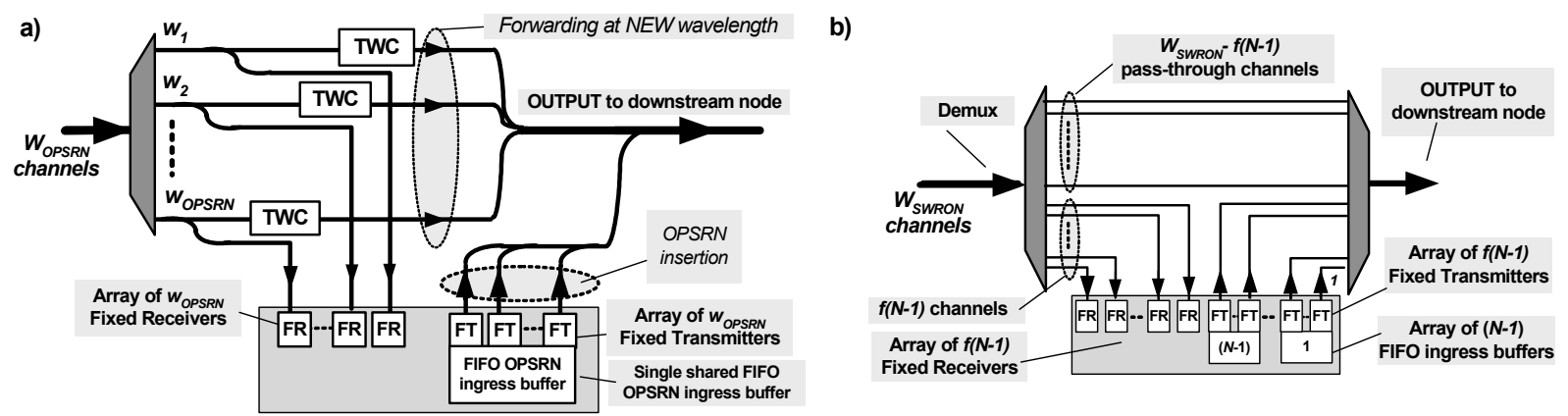

Figure 2. Node architectures for the a) OPSRN, and b) SWRON.

\subsection{Optical Packet Switched Ring Network Architecture}

OPSRNs constitute a long-term solution to bridge the widening "metro-gap" [1]. They benefit from attractive properties of optical and electronic technologies, by combining cost-effective WDM transmission and flexible electrical buffers to store packets from the AN in ingress buffers until insertion on the ring is desirable. A number of OPSRN architectures have been proposed. To support asynchronous operations with Variable Length Packets (VLP) without segmentation under dynamically varying unbalanced traffic scenarios, this study considers our recently proposed OPSRN node architecture. It employs the associated Asynchronous Insertion Priority Scheduling with Insertion Threshold (AIPSwIT) MAC protocol [3, 4], which works in the following way:

Packets arriving from an AN attached to a MAN ring node, are stored in an OPSRN ingress module, which is a single shared FIFO buffer. The AIPSwIT MAC controls the insertion process, and starts inserting the oldest packet on an idle wavelength via the corresponding FT, when more than $W_{F R E E}$ wavelengths are free. The idle wavelength is arbitrarily chosen among the $W_{O P S R N}$ wavelengths available; the node has full accessibility to the OPSRN bandwidth. Contention between packets to be forwarded and packets being inserted on the ring is resolved using a TWC to convert the forwarded packet to an idle OPSRN wavelength. There is a high chance of finding such a free wavelength, when the AIPSwIT MAC insertion threshold, $W_{F R E E}$, is properly chosen and the network is well dimensioned. However, if contention cannot be resolved, the forwarded packet is lost. Packets can be received at all wavelengths at all nodes, enabling multicast (not studied here). When a packet reaches its destination, the TWC is used as an optical gate to prevent propagation to downstream nodes, thereby enabling spatial wavelength reuse, similar to the use of SOAs for this purpose in slotted MACs [5]. As detailed in section 3.2, packets are discarded from the ingress buffer when buffer capacity is surpassed, or when a time-out occurs.

This OPSRN architecture results in a bandwidth efficient OPSRN, which also supports fairness for unbalanced traffic, by operating with different values of $W_{F R E E}$ in the network nodes. This comes at the expense of using a relatively high number of sub-wavelength granularity switches (TWCs), as discussed in section 4 . Note that instead of using $W_{O P S R N}$ FTs per node, one can obtain the same performance using a reduced number of Tuneable Transmitters (TTs) when ensuring that the TT count is sufficiently high to prevent buffer overflow caused by lack of transmitters [4]. This option should be included in a cost analysis of the network before implementation. However, this work assumes that all nodes are equipped with $W_{O P S R N}$ FTs and FRs, to avoid imposing any limitation on unbalanced traffic scenarios, and to enable a fair hardware comparison between the different architectures.

\subsection{Wavelength Routed Optical Network Architecture}

Static- and Dynamic Wavelength Routed Optical Network (DWRON and SWRON) architectures do not offer any statistical multiplexing gain, but can be realised as Optical Circuit Switched (OCS) network with transfer guarantees [7]. DWRONs can be reconfigured on a slow ( $>\mathrm{ms})$ time-scale using Reconfigurable Optical Add/Drop Multiplexers (ROADM), whilst SWRONs use Static OADMs, e.g. multiplexers and demultiplexers, to passively guide optical data from its $n_{S}$ to its $n_{D}$, based on its input wavelength. This study maximises the simplicity advantage of WRONs compared to OPSRNs, by only considering SWRONs.

With $N$ nodes, the SWRON needs $N(N-1)$ connections for full connectivity, which can be offered using $N(N-1) / 2$ wavelengths per fibre, when all destinations employ spatial wavelength reuse. One can increase the 
capacity between each $\left(n_{S}, n_{D}\right)$ pair by an integer factor $f$, by increasing the SWRON wavelength count correspondingly, i.e. $W_{S W R O N}=f \times x N(N-1) / 2$.

Figure $1 \mathrm{~b}$ illustrates the SWRON node architecture. Each node has an ingress module, consisting of $N-1$ FIFO buffers, one per destination node, each associated with $f$ FTs. The node also has $f \mathrm{x}(N-1)$ FRs to receive the data terminated in this node. This architecture completely isolates the performance of the different $\left(n_{S}, n_{D}\right)$ pairs. This makes the network completely fair for uniform traffic, but prevents any sharing of network resources.

\subsection{Hybrid Architectures}

Hybrid architectures for an optical core mesh network have been proposed to combine the efficiency and flexibility of packet switching and the simplicity and maturity of circuit switching.

The ORION (Overspill Routing In Optical Networks) concept aims at overcoming temporarily overload situations by combining a DWRON and an electrically switched point-to-point WDM network [6], effectively sharing the same bandwidth. When a packet cannot be forwarded on an already established lightpath from its $n_{S}$ to its $n_{D}$, it enters the so-called "overspill" mode, which consists of using unused capacity in lightpaths leading to other destinations via the next link. At the next-hop node, the overspill packet is detected, extracted (using a 1x2 optical switch), and $\mathrm{O} / \mathrm{E}$ converted, before again using free capacity on existing lightpath on the next link.

It has also been proposed to support a Guaranteed Service (GS) and a Best-Effort (BE) CoS on the same WDM channels, by transmitting packets from the two $\mathrm{CoS}$ on orthogonal polarisations [7]. After segregation at the input [8], the two CoS are switched using a SWRON and an OPS switch matrix, respectively.

This work complements and extends our recent hybrid architecture proposal combining SWRON and OPSRN node architectures for an optical switched ring MAN [9]. The target is to obtain an attractive balance between performance (including overall throughput and fairness, for unbalanced traffic) and complexity (in terms of optical hardware and electronic scheduling). The hybrid aspect is achieved by allowing packets to be handed over from the SWRON- to the OPSRN electrical ingress buffers, assuming a single CoS. The OPSRN part provides an optical solution to support quite different traffic scenarios without reconfiguring the WRON. This enables use of a SWRON, being simpler than a DWRON. Furthermore, the OPSRN part offers statistical multiplexing gains and is suitable for bursty traffic. The handover takes place when overflow or timeout occurs in a SWRON FIFO ingress buffer, as detailed in section 3.2. Hence, packets can only be lost when discarded from the OPSRN ingress buffer or during forwarding by the OPSRN.

This hybrid architecture is fundamentally different from previous hybrid architectures, since the WDM bandwidth of $W$ channels is separated into a SWRON- and an OPSRN waveband, containing $W_{S W R O N}$ and $W_{O P S R N}$ channels, respectively. This enables use of WDM- and switching equipment adapted to each architecture within each waveband, and avoids detection and segregation of different types of optical data at node interfaces, as well as use of polarisation multiplexing. Complexity related to the handover occurs only in electronics at the ingress node, avoiding multiple $\mathrm{O} / \mathrm{E} / \mathrm{O}$ conversions, and enabling a bounded delay-jitter, as detailed in section 3.2 .

\section{NETWORK STUDY}

\subsection{Scope}

This work studies performance with a fixed total network input load of $1.28 \mathrm{Tbit} / \mathrm{s}$ assuming $10 \mathrm{Gbit} / \mathrm{s}$ channel bitrates. The network has $N=8$ nodes, and the mean hop count is $H=N / 2=4$ hops in all traffic scenarios. Network utilisation (of WDM bandwidth) is then given by $\alpha=64 / W$, meaning that $W=64$ corresponds to $100 \%$ utilisation. In practice, $W$ is somewhat higher, depending on the desired performance, the input traffic and the network architecture. The dimensioning study in section 3.3 identifies the required $W$ to reach a certain performance threshold, for Poisson and bursty input traffic with a uniform traffic scenario. The study in section 3.4 evaluates the effect of unbalanced traffic, for two dimensioning cases.

Each scenario is studied by optimising the AIPSwIT insertion thresholds. In real operation, this should be carried out by a low-dynamic network central manager [4]. However, the MAC protocol is still distributed, in the sense that all high-dynamic scheduling decisions are made independently by the schedulers within the node in question, based on the MAC protocol and associated parameters, provided by the network management. Performance during transient periods between different stable scenarios is out of the scope of this study.

\subsection{Simulation study and input parameters}

Performance is studied by OPNET simulations for asynchronous arrival and exponential packet length distribution. The "bursty" traffic scenario is modelled by a large number of independent Pareto traffic sources, with a Hurst parameter of $0.8 .95 \%$ confidence intervals are calculated using 10 independent simulation runs, ignoring the transient period. The link propagation delay is set to $25 \mu \mathrm{s}$ ( $5 \mathrm{~km}$ link distance). The ring propagation time is constant for each $\left(n_{S}, n_{D}\right)$ pair, whilst the ingress buffer delay may vary between packets. Hence, limiting the maximum time spent in the buffer, $D$, limits the maximum delay and the maximum delay 
variation between consecutive packets, reducing complexity and size of egress node reordering buffers [3]. Furthermore, the size of the ingress buffers should be limited to enable simple ingress buffers storage hardware and scheduling units. In this study, the OPSRN FIFO ingress buffer in each node can contain up to $B_{O P S R N}$ packets, before overflow occurs and the oldest packet is discarded. Moreover, each packet can only reside in the buffer for $D_{O P S R N}$ MPD, before being discarded. All $N-1$ SWRON FIFO buffers in a node can contain up to $B_{\text {SWRON }}$ packets and each packet can only reside in the buffer for $D_{\text {SWRON }}$ MPD, before being either discarded (in the SWRON architecture) or handed over to the OPSRN (in the hybrid architecture). For the hybrid architecture, the total buffer capacity of a node becomes $B=(N-1) B_{S W R O N}+B_{O P S R N}$, and the maximum delay becomes $D=D_{\text {SWRON }}+D_{\text {OPSRN }}$. For a fair comparison, total buffer resources were fixed at $D=8 \mathrm{MPD}$ and $B=112$ packets. For the hybrid architectures, total buffer capacity and delay were evenly distributed between the SWRON- and OPSRN part, i.e. $B_{O P S R N}=56, B_{S W R O N}=8$ and $D_{S W R O N}=D_{O P S R N}=4 \mathrm{MPD}$.

Network performance is expressed by the Relative Throughput (RT) and the Fairness Index (FI) [4]. RT is the ratio between the overall number of packets successfully received at its correct destination and offered to a source. For $R T$ close to unity, the absolute throughput of network almost equals the input load of $1.28 \mathrm{Tbit} / \mathrm{s}$. From a QoS aspect, PLR is however more important, and it is given by $P L R=1-R T$.

$F I$ gives a more detailed view of performance, by quantifying how the relative throughput between different $\left(n_{S}, n_{D}\right)$ pairs, $R T\left(n_{S}, n_{D}\right)$, vary. The objective is to obtain "relative" fairness, i.e. an $R T\left(n_{S}, n_{D}\right)$ independent of the load of the $\left(n_{S}, n_{D}\right)$ pairs, since it is assumed that this load is agreed upon between AN and MAN operators. When this is achieved, the user in large AN (resulting in a higher than average input load of the MAN ring node) will not be penalised compared to users in small ANs, and the $R T$ of different $\left(n_{S}, n_{D}\right)$ pairs is almost equal. This is quantified using our adaptation of the "Fairness Index" proposal of Jain [10], proposed in [4]. FI is calculated using the $R T\left(n_{S}, n_{D}\right)$ of all $z=N(N-1)$ connections, cf. Equation (1). The $F I$ is bounded by 0 (completely unfair) and 1 (completely fair). FI should be very close to unity $(>0.999)$, before we intuitively would describe it as "fair", as graphically illustrated in [4].

$$
F I=\frac{\left(\sum_{i=1}^{i=z} \mathrm{RT}\left(\mathrm{n}_{\mathrm{S}}, \mathrm{n}_{\mathrm{D}}\right)_{i}\right)^{2}}{z\left(\sum_{i=1}^{i=z} \mathrm{RT}\left(\mathrm{n}_{\mathrm{S}}, \mathrm{n}_{\mathrm{D}}\right)_{i}^{2}\right)} .
$$

\subsection{Uniform Traffic Performance}

For Poisson traffic, the SWRON achieves $R T=0.998$, when $f=3$, i.e. $W=W_{S W R O N}=84$, as illustrated by the single-point white square in Fig. 3a. We set this as the network dimensioning performance target, corresponding to a mean network PLR of $2 \times 10^{-3}$. Figure 3 a also shows $R T$ vs. $W$ for the other architectures, both for Poisson (white symbols) and Pareto traffic (grey symbols).

For this uniform traffic scenario, $F I$ is omitted, since the network is very fair when $R T$ is sufficiently high ( $F I>0.99999$ for $R T>0.998$, for all architectures). Only for low values of $R T$, transfers with long hop count are discriminated in the OPSRN part, since priority is given to packets being inserted [4].

To reach the target $R T$ of 0.998 , the SWRON, Hybrid_f2, Hybrid_f1, and OPSRN architectures require values of $W$ of 84, 79, 84 and 73, respectively. This corresponds to values of $W_{O P S R N}$ of $0,23,56$ and 73, taking the values of $W_{\text {SWRON }}$ of $84,56,28$ and 0 into account. Neither of the architectures needs more than a $5 \%$ increase in wavelength count to offer same performance for bursty traffic.

The OPSRN is thus the more bandwidth efficient, whilst SWRON is the least efficient. This can be expected since the statistical multiplexing gains are the highest for the OPSRN, and the lowest for the SWRON. The Hybrid_f2 architecture benefits from statistical multiplexing gains in its OPSRN part to outperform the SWRON architecture. However, the Hybrid_fl requires the same $W$ as the SWRON to reach the target $R T$. This is attributed to the fact that both OPSRN and SWRON can fully utilise their buffer resources, whilst the somewhat arbitrarily design choice of sharing buffer resources equally (i.e. $B_{O P S R N}=56, B_{S W R O N}=8$ and $D_{\text {SWRON }}=D_{\text {OPSRN }}=4 \mathrm{MPD}$ ) is not necessarily the optimum for the hybrid designs, depending on the architecture and $W$. It turns out that Hybrid_f1 in particular suffers from this, and further improvements can be achieved by changing the distributions of buffer resources. E.g. adjusting to $D_{S W R O N}=2 \mathrm{MPD}$ and $D_{O P S R N}=6 \mathrm{MPD}$ enables also the Hybrid_f1 to reach the target RT for $W=79$.

\subsection{Unbalanced Traffic Performance}

Performance is then studied for unbalanced traffic, modelled by an asymmetric server scenario [4]. The Server Relative Load Factor $(S R L F)$ denotes the ratio of traffic originating at the server (e.g. node \#1) relative to that from each of the other nodes, with $S R L F=1$ corresponding to uniform traffic. The asymmetric server scenario is very demanding in ring networks, since in particular the link downstream of the server will be heavily loaded, even overloaded for OPSRNs with sufficiently high SRLF [4]. For the SWRON, the wavelengths from the 
server are overloaded for small values of $S R L F$, since the limited accessibility prevents the server from benefiting from the reduced load of other wavelengths. Note that performance does not depend on which node is the server. However, the results will much depend on the dimensioning, and we study two cases:

- $\quad D I M_{W_{-} M I N}$ case: Uses the value of $W$ that enabled $R T$ of 0.998 for each architecture for uniform, Poisson traffic, i.e. SWRON, Hybrid_f2, Hybrid_f1, and OPSRN have $W$ of 84, 79, 84 and 73, respectively.

- $\quad D_{W_{-} F I X 84}$ case: All architectures use $W=84$.

In all cases, the $A I P S w I T$ insertion thresholds are optimised for each $S R L F$ value, by allowing a different $W_{F R E E}$ value at the server, compared to the other nodes. Note that further gains in performance may be achieved by optimising all $N$ insertion thresholds individually. However, priority is here given to simple network operation. Figure $3 \mathrm{~b}-3 \mathrm{~d}$ shows $R T$ and FI vs. SRLF for the different architectures for these two dimensioning cases. One observes the following:

- The SWRON architecture poorly supports unbalanced traffic: lack of bandwidth sharing and lack of FT/FR sharing leads to overload of wavelengths between $\left(n_{S}, n_{D}\right)$ pairs with much traffic.

- The OPSRN performs much better, but suffers from its high utilisation in the DIM $M_{W_{-} M I N}$ case, since the low $W$ quickly induces overload of the link after the server. For the DIM $W_{-} F I X 84$ case, an SRLF of 2 can be supported with a higher $R T$ and FI than the performance target both for Poisson and bursty traffic. However, an $S R L F$ of 4 only can be supported for an $R T$ of 0.990 and a FI of 0.99992.

- The hybrid architectures achieve an improved $R T$ and FI, compared to the SWRON. In the DIM $M_{W_{-} M I N}$ case, the higher $W$ of the Hybrid_fl architecture makes it outperform the Hybrid_f 2 architecture. When using the same channel count (in the DIM $M_{W F X 84}$ case), the Hybrid f1 benefits from a quite high bandwidth sharing, to almost match the OPSRN performance for SRLF up to 4 (RT of 0.997 and 0.989 for $S R L F$ of 2 and 4). In contrast, the Hybrid f2 architecture suffers from limited bandwidth sharing for high $S R L F$, with $R T$ values of 0.998 and 0.951 . Note that the Hybrid_f1 suffers slightly more from bursty traffic than the OPSRN architecture.

a) $R T$ vs. $W$ for different network architectures, for Uniform traffic.

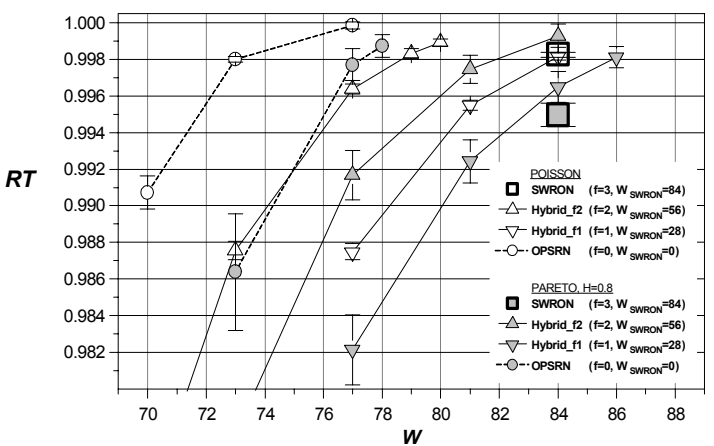

C) $R T$ and $F I$ vs. SRLF for Poisson traffic for $D I M_{W_{-} F X 84}$ scenario.

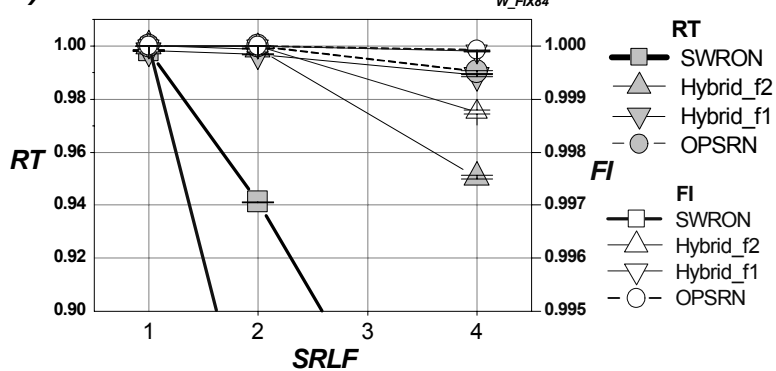

b) $R T$ and $F I$ vs. SRLF for Poisson traffic for $D I M_{W_{-} M I N}$ scenario.

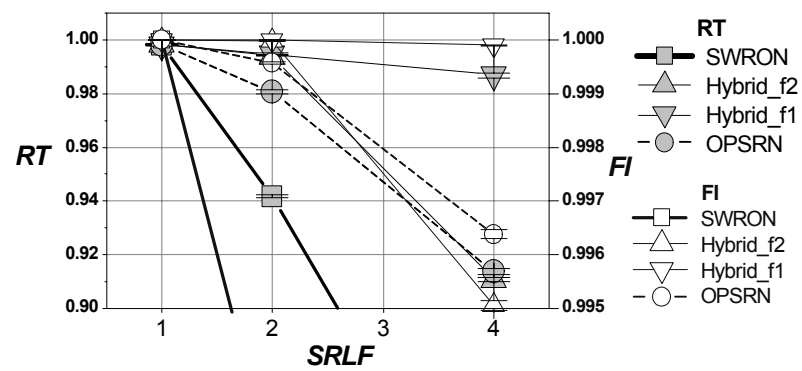

d) $R T$ and $F I$ vs. SRLF for bursty traffic for $D I M_{W_{-F} \times 84}$ scenario.

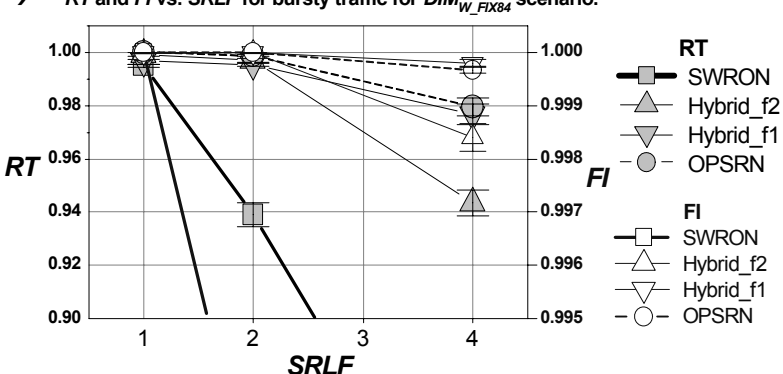

Figure 3. a) RT vs. W, for the proposed architectures. b) - d) Performance for unbalanced traffic scenarios, for $D I M_{W_{-} M I N}$ and DIM $M_{W_{-} F I X 84}$ dimensioning. 


\section{DISCUSSION}
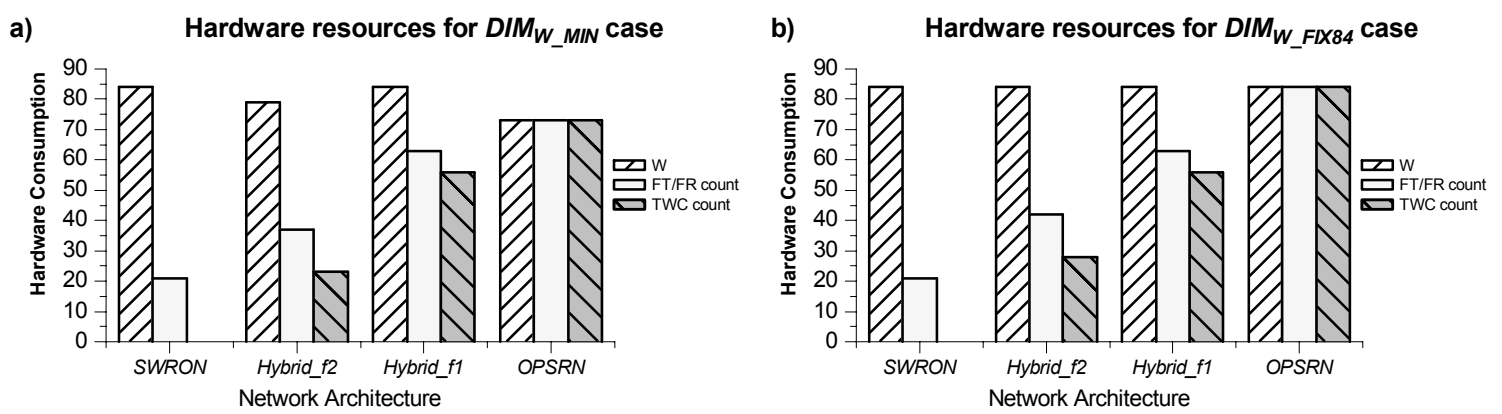

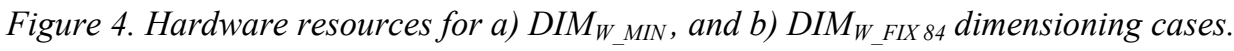

The hardware aspect is important to evaluate the attractiveness of the proposed architectures. Figure 4 quantifies the total WDM channel count, $W$, the FT and FR count per node, and the TWC count per node, both for the $D I M_{W}$ MIN and the DIM ${ }_{W}$ FIX84 4 case.

Figure 4a shows that the OPSRN has the lowest $W$ to reach the performance target, at the expense of the highest FT/FR- and TWC count. This utilisation of $0.88 \%$ is achieved thanks to high statistical multiplexing gains. Hybrid_f2 represents a potential utilisation-complexity trade-off. Still, for uniform traffic, the SWRON seems the more attractive solution, by avoiding use of TWCs and by minimising FT/FR count. However, one should keep in mind that the choice of a $R T$ performance target that corresponded exactly to the $R T$ of the SWRON for an integer $f$ somewhat favours this architecture. E.g. increasing the target RT or load slightly would require adding a whole set of 28 new WDM channels, which would reduce SWRON utilisation from $76 \%$ to $57 \%$. The OPSRN and the hybrid architectures have a finer dimensioning granularity, and are thus more flexible.

Keeping the results of section 3.4 in mind, Fig. $4 \mathrm{~b}$ indicates that the excellent performance of the OPSRN is achieved at the expense of extensive use of TWCs and FT/FRs. Since the Hybrid_f1 architecture had very similar performance, and reduces the FT, FR and TWC count by $\sim 25 \%$, it seems more attractive. If $S R L F$ is maintained at 2 or lower, the Hybrid f2 offers near similar performance for a hardware count reduction of $\sim 60 \%$. As discussed in section 3.3, the hybrid architectures could further improve performance by optimising the distribution of buffer resources.

\section{CONCLUSIONS}

The OPSRN combines spatial wavelength reuse and full bandwidth accessibility to achieve very high bandwidth efficiency and good support of dynamic traffic patterns. This comes at the expense of using a high number of TWCs, FTs and FRs. The SWRON, on the other hand, minimises the hardware complexity, but does not support dynamic, unbalanced traffic very well. Hybrid architectures were identified as attractive trade-offs for unbalanced traffic; the choice of hybrid architecture depends on the input traffic and the cost of active components.

\section{REFERENCES}

[1] M. Herzog, M. Maier, M. Reisslein: Metropolitan area packet-switched WDM networks: a survey on ring systems, IEEE Communications Surveys, 6(2), pp. 2-20, Second Quarter 2004.

[2] S. Yao, S.J.B. Yoo, B. Mukherjee, S. Dixit: All-optical packet switching for metropolitan area networks: opportunities and challenges, IEEE Communications Magazine, 39(3), pp. 142-148, 2001.

[3] M. Nord, S. Bjørnstad, M. L. Nielsen: Distributed MAC Protocol for Optical Packet Switched Ring Network Supporting Variable Length Packets, OSA J. on Opt. Netw., 4(4), pp. 213-225, 2005.

[4] M. Nord: Fairness Support in Flexible Asynchronous Optical Packet Switched Ring Networks, submitted to Elsevier Optical Switching and Networking, 2005.

[5] C. Develder, R. V. Caenegem, E. Baert, M. Pickavet, P. Demeester: Active versus passive OPS architectures for metro rings: network dimensioning point of view, in Proc. ECOC 2003, We 1.4.4.

[6] E. Van Breusegem et al.: A broad view on overspill routing in optical networks: a real synthesis of packet and circuit switching?, Elsevier Optical Switching and Networking, vo1. 1, pp. 51-64, 2005.

[7] S. Bjornstad, D. R. Hjelme, N. Stol: A highly efficient optical packet switching node design supporting guaranteed service, in Proc. ECOC 2003, paper Mo.4.4.5.

[8] M. Nord, S. Bjørnstad, M. L. Nielsen, B. Dagens: Demonstration of optical packet switching scheme for header-payload separation and class-based forwarding, Techn. Digest OFC 2004, vol. 1, pp. 563-565.

[9] M. Nord: Hybrid Optical Ring Network Architectures for the MAN, submitted to ECOC 2005.

[10] R. Jain, D. Chiu, W. Hawe: A quantitative measure of fairness and discrimination for resource allocation in shared computer systems, Digital Equipment Corporation DEC-TR-301, Sept. 1984. 